\title{
Angiopoietin-2: Development of Inhibitors for Cancer Therapy
}

\author{
Bo Hu, PhD and Shi-Yuan Cheng, PhD
}

\begin{abstract}
Angiopoietin-2 (Ang2) is a member of the Ang family, which plays an important role in angiogenesis during the development and growth of human cancers. Ang2's role in angiogenesis generally is considered as an antagonist for Ang1, inhibiting Ang1-promoted Tie2 signaling, which is critical for blood vessel maturation and stabilization. Ang2 modulates angiogenesis in a cooperative manner with another important angiogenic factor, vascular endothelial growth factor A. Genetic studies have revealed that Ang2 also is critical in lymphangiogenesis during development. However, new evidence suggests more complicated roles for Ang2 in angiogenesis in physiologic processes and invasive phenotypes of cancer cells during progression of human cancers. This article discusses recent studies of Ang2 in angiogenesis and the implication of Ang2 as a therapeutic target as well as a potential inhibitor for antiangiogenesis treatment for cancer patients.
\end{abstract}

\section{Introduction}

It has been established that growth and progression of human tumors depend on pathologic growth of new blood vessels (neoangiogenesis). Angiogenesis generally refers to the growth of vessel sprouts from capillary blood vessels, including endothelial cell (EC) migration toward stimuli in the microenvironment, EC proliferation, tube formation, and intussusception, followed by recruitment of peri-ECs and synthesis of extracellular matrix (ECM) [1]. The process of tumor angiogenesis is orchestrated by an array of angiogenic stimulators and inhibitors. Within the tumor microenvironment, tumor cells and tumor-associated stromal cells, such as macrophages and other immune cells, produce angiogenic factors such as vascular endothelial growth factor (VEGF) and endogenous inhibitors such as thrombospondin-1 [1]. Angiopoietins (Ang), a family of angiogenic factors, have distinct functions in tumor angiogenesis and behavior. One Ang family member in particular, Ang2, has been the focus of studies not only because of its unique roles in modulating angiogenesis, but also because of some of its unconventional functions; the close link of Ang2 expression to tumor progression, growth, and invasion/metastasis; and its great potential as a therapeutic target, a prognostic marker, and an inhibitor of human cancers. This article reviews recent literature describing this special growth factor.

\section{Ang2's Roles in Angiogenesis}

\section{Ang2 in physiologic angiogenesis and lymphangiogenesis}

The Ang family has four members-Ang1, Ang2, Ang3, and Ang4—and two related receptors, Tie2 and Tie1 [2•]. In vivo, Ang1 and Ang2 have complementary functions in maintaining vascular function and integrity. Ang1 maintains the integrity of vasculature by recruiting peri-

\footnotetext{
Copyright $\odot 2009$ by Current Medicine Group LLC

Corresponding author, Shi-Yuan Cheng, PhD, Cancer Institute and Department of Pathology, University of Pittsburgh, HCCLB, 2.26f, 5117 Centre Avenue, Pittsburgh, PA 15213, USA. chengs@upmc.edu.

Disclosures

No potential conflicts of interest relevant to this article were reported.
} 
ECs into blood vessels [1]. Transgenic mice deficient in Ang1 had disrupted interactions between ECs and peri-ECs that severely impaired vascular function, leading to embryonic death of the mice $[3,4]$. Transgenic mice overexpressing VEGF-A in the skin induced hyperpermeable vessels. Moreover, vessels in Ang1-overexpressing mice were not leaky and were resistant to leaks caused by inflammatory agents [5]. Coexpression of Ang1 and VEGFA had an additive effect on angiogenesis and resulted in leakage-resistant vessels, typical of Ang1-expressing vessels [6].

Initially, Ang2 was identified as an antagonist for Ang1 by inhibiting Ang1 activation of Tie2. Transgenic mice overexpressing Ang2 had phenotypes similar to those of Ang1-null mice, displaying disrupted interactions between ECs and peri-ECs that severely disrupted blood vessel formation in mouse embryos. In adult mice and humans, Ang2 is expressed only at the sites of vascular remodeling [7]. Ang2's major role in angiogenesis appears to be in cooperation with VEGF-A function. In the presence of VEGF-A, Ang2 promotes vascular sprouting and destabilizes blood vessels by disrupting interactions between ECs and peri-ECs, thus enhancing VEGF-A stimulation. In the absence of VEGF-A, however, Ang2 acts as a suppressor that accelerates vessel regression [8]. This hypothesis was validated by a study using a model of a transient ocular microvessel network that surrounds the anterior part of the lens in the developing eye. In the presence of exogenous VEGF-A, Ang2 promoted a rapid increase in capillary diameter, remodeling of the basal lamina, and proliferation and migration of ECs and stimulated the sprouting of new blood vessels. When VEGF was inhibited in the system by soluble VEGF receptor-1, Ang2 induced EC apoptosis, resulting in vessel regression [9].

Ang2's role in developmental angiogenesis was further investigated by genetic knockout studies. Unexpectedly, mice lacking Ang2 exhibited major lymphatic vessel defects with no effect on vascular development. At birth, the vasculature in newborn Ang2-null pups was indistinguishable from that in wild-type pups. However, postnatal Ang $2^{-1-}$ mice had dramatic defects in subsequent vascular remodeling. In the eye, the hyaloid vasculature normally had largely regressed in wild-type and heterozygous mice by postnatal day 10. In contrast, the hyaloid vasculature was maintained aberrantly in Ang2-deficient mice at this age. This and other postnatal defects in the vasculature of Ang2-null mice revealed that Ang2 is absolutely required for the coupled vascular regression and sprouting events that comprise normal angiogenic remodeling of the ocular vasculature. Genetic rescue with Ang1 corrected the lymphatic but not the postnatal angiogenesis defects, suggesting that Ang2 acts as a Tie2 agonist in the former setting but as an antagonist in the latter [10]. Two new studies provide further details about these phenomena. In Ang2-deficient mice, lymphatic vessels fail to mature and do not exhibit a collecting vessel phenotype. Dermal lymphatic vessels in Ang2-null pups prematurely recruit smooth muscle cells and do not undergo proper postnatal remodeling. However, genetic rescue with Ang1 in Ang2-null mice restores a normal hierarchic lymphatic structure, suggesting that Tie2 activation is required for normal lymphatic development [11]. In wild-type mice, Ang2 exerts a dual role in scheduled hyaloid vascular EC death (vessel regression) in the eye. Ang1 stimulation of Tie 2 activates Akt signaling and suppresses death of hyaloid vascular ECs. In contrast, Ang2 induces hyaloid vascular EC death by suppressing Akt in ECs and upregulates macrophage Wnt7b, a Wnt receptor ligand that associates with hyaloid vessels. Wnt7b stimulates cell cycle entry of vascular ECs and couples cell death. Because this second pathway is macrophage derived and Wnt7b dependent, genetically engineered Ang1 could not rescue the postnatal angiogenesis defects in Ang2-deficient mice [12]. These observations also explain sustained hyaloid vasculature in the eyes of Ang2deficient mice [10].

In vitro, Ang2 was shown to be an antagonist and agonist for Tie2-mediated signaling, enhancing EC migration, survival, and tubelike structure formation [2,13]. Moreover, stimulated release of endogenous Ang2 or overexpression of Ang2 in ECs destabilizes 
quiescent ECs through an internal autocrine loop, leading to EC detachment and a vessel regression phenotype in vitro and ex vivo. Such a perturbation could be rescued by introducing exogenous Ang1 and VEGF-A, but not a soluble Tie2 [14]. Ang2 also cooperates with Ang1 in modulating endothelial development from circulating cord blood $\mathrm{CD} 34^{+}$progenitors. Endogenous Ang1 regulates initial EC commitment of the $\mathrm{CD} 34^{+}$progenitor cells, whereas Ang2 contributes to expansion of the endothelial progeny [15]. Additionally, combining Ang2 and Ang1 directly activates ECs and neutrophils to promote a proinflammatory response through Tie2- and $\beta_{2}$ integrin-mediated signaling [16].

\section{Ang2 in tumor angiogenesis}

Ang2's role in tumor angiogenesis has been controversial [2•]. Upregulation of Ang2 correlates with the malignancy of various types of human cancers. Ang2 overexpression in various types of human cancer cell lines augmented tumor angiogenesis and growth in mice. In contrast, other studies reported that specific induction of Ang2 in gliomas, mammary carcinomas, and lung carcinomas inhibited tumor growth and metastasis [2•,17]. Systemic Ang2 overexpression led to unexpected massive tumor vessel regression within 24 hours, even without concomitant VEGF inhibition. By impairing pericyte coverage of the tumor vasculature, Ang2 induced massive tumor vascular regression while improving perfusion in surviving tumor vessels and transiently exacerbating tumor hypoxia, without affecting adenosine triphosphate levels. This significantly inhibited tumor angiogenesis, promoted tumor apoptosis, and suppressed tumor growth [18•]. Ang2 was shown to prevent ECs from forming a tubelike structure in vitro and to impair angiogenesis in a chorioallantoic membrane chicken model system. In a glioma xenograft model, sustained Ang2 expression disrupted blood vessel formation and inhibited glioma growth in mouse brain [19].

In addition to Ang2's role in tumor angiogenesis, its expression by tumor cells has been linked to invasive and metastatic phenotypes of gliomas and gastric, colon, prostate, and breast cancers [20,21 •]. Recently, we reported that Ang2 is co-upregulated with matrix metalloprotease (MMP)-2, membrane type-1 MMP, and laminin- $\gamma-2$ in invasive areas but not in the central regions of clinical primary glioma specimens. Statistical analysis revealed a significant link between the preferential expression of these molecules and invasiveness [22]. Consonant with these features, intracranial xenografts of U87MG glioma cells engineered to express Ang2 were highly invasive into adjacent brain parenchyma compared with isogenic control tumors. In regions of Ang2-expressing tumors actively invading the brain, high levels of MMP-2 expression and increased angiogenesis also were evident. A link between these two features became apparent when stable Ang2 expression by U87MG cells or treatment of several glioma cell lines with recombinant Ang2 in vitro caused MMP-2 activation and increased invasiveness. Conversely, MMP inhibitors suppressed Ang2-stimulated activation of MMP-2 and Ang2induced cell invasion [23]. Our observations of Ang2 promotion of glioma cell invasion were supported by other studies in gliomas and gastric cancers and by our own results using various breast cancer xenograft models in animals [20,21•].

\section{Ang2 Signaling and Modulation}

\section{Tie2-mediated signaling}

Ang1 and Ang2 interact with their cognate receptor, Tie2, which is most frequently expressed in ECs. Upon binding Angs, the Tie2 receptor dimerizes and becomes phosphorylated in tyrosine residues at its C-terminal [24]. Activated Tie2 stimulates phosphatidylinositol 3kinase (PI3K)-dependent activation of Akt, leading to increases in cell survival, suppression of caspase 3,7, and 9 activity, and attenuation of mitochondrial-derived activator of caspase (Smac) release. Ang1 stimulates Tie2, activating the DokR-Pak, Nck, and Grb14 pathways; inhibiting EC proliferation; and displaying an antiangiogenic property [13]. Ang1 also 
upregulates cyclin D1 expression and activates mitogen-activated protein kinase (MAPK) and PI3K in murine IBE and human umbilical vein endothelial cells [25]. In addition, COMPAng1-induced vascular enlargement and blood flow are mediated mainly through Tie2 activation, and sustained Tie 2 overexpression may participate in the maintenance of vascular changes [26]. The protective role of ECs from apoptosis by Ang1 or in synergy with VEGFA has been widely observed in vitro, in vivo, and under various stress conditions [2•,17]. Ang2 has been found to be an antagonist as well as an agonist for Tie2 receptor signaling in modulating EC migration and survival [13]. However, unexpected data of Ang2 function in ECs have been documented. For example, Harfouche and Hussain [27] demonstrated that treatment of ECs with Ang2 (150-300 ng/mL) increases the phosphorylation of Tie2, Akt, extracellular signal-regulated kinase 1/2 (ERK1/2), and p38 members of MAPKs but inhibits JNK phosphorylation. When ECs were stimulated with a combination of Ang2 and VEGF, ERK1/2 activation also was inhibited. In contrast, augmentation of Ang1-induced ERK1/2 phosphorylation was triggered by VEGF treatment. Moreover, Ang2 treatment did not stimulate cell migration in vitro but significantly attenuated serum deprivation-induced EC apoptosis and promoted cell survival. In non-ECs that were found to express Tie2, such as human glioma cell lines, Ang2 inhibited VEGF-A expression at the mRNA and protein levels in Tie2-expressing glioma cells but not in Tie2-negative tumor cells. Ang2 regulated VEGF expression at the transcriptional level in relation to a decrease in hypoxia-inducible factor (HIF)-1 $\alpha$ expression and HIF-DNA-binding activity. Tie2 silencing by small interfering RNA (siRNA) rescued the Ang2-mediated down-modulation of VEGF, suggesting an essential role for Tie2 in this regulatory loop [28].

\section{Interactions with integrins}

Although Ang2 modulates angiogenesis through interaction with the Tie2 receptor [29], it contains a highly conserved $\mathrm{N}$-terminal fibrinogen-like receptor-binding domain that may form a functional association with certain integrin receptors [2•]. Ang1 and Ang2 were shown to stimulate Tie2-independent cell adhesion of ECs and fibroblasts to Ang1- or Ang2-coated surfaces through $\alpha_{5} \beta_{1}$ and $\alpha_{\mathrm{v}} \beta_{5}$ integrin-mediated activation of ERK and focal adhesion kinase (FAK) signaling [30]. Skeletal myocytes lacking Tie2 adhere to Ang1- and Ang2-coated surfaces in a similar manner as to laminin, fibronectin, and vitronectin. The Ang-stimulated skeletal myocyte adhesion is mediated by integrin receptors such as $\alpha_{5} \beta_{1}$, activating ERK and FAK signaling and promoting cell survival [31]. It has been postulated that Ang's interaction with integrins likely occurs through the fibrinogen-like receptor-binding domain present in the Ang protein structure [32]. This theory was examined recently, showing that a monomeric Ang1 variant ( $\triangle$ Ang), composed only of the fibrinogen-like receptor-binding domain that also exists in Ang2, ligates Tie2 without activating the receptor. Moreover, $\Delta$ Ang binds to $\alpha_{5} \beta_{1}$ integrin with an affinity similar to that of Tie2. When ECs were plated on $\triangle$ Ang-coated surfaces, $\triangle$ Ang displayed biologic effects similar to those of the full-length Ang1, stimulating cell adhesion, ERK signaling, and vascular maturation [33].

Modulation of EC function and angiogenesis by Ang1 through direct interaction with integrins also was demonstrated in ECs expressing the Tie 2 receptor. Immobilized Ang 1 was shown to selectively mediate $\alpha_{5} \beta_{1}$ integrin out-side-in signaling, leading to cross-talk between Tie 2 and $\alpha_{5} \beta_{1}$ and promotion of angiogenesis [34]. In tumor cells lacking Tie 2 expression, we showed that Ang2 induces glioma cell invasion by stimulating MMP-2 expression through the $\alpha_{\mathrm{v}} \beta_{1}$ integrin and FAK pathways [35•]. We also reported that Ang2 associates with $\alpha_{5} \beta_{1}$ integrin in Tie2-deficient breast cancer cells. Ang2 activates an integrin-mediated signaling pathway, leading to breast cancer cell invasion and metastasis. Inhibition of $\beta_{1}$ or $\alpha_{5}$, but not other integrins, attenuates Ang2 modulation of integrin-linked kinase (ILK), Akt, glucose synthase kinase-3 $\beta$, Snail, E-cadherin, and vimentin, and Ang2-stimulated breast cancer cell motility $[21 \bullet]$. Furthermore, similar to the association of Ang2 with integrins in skeletal myocytes 
[31] and of PG-MV/vesican with integrin in glioma cells [36], the association of Ang2 with integrins was highly calcium and manganese dependent in our system. These studies established Ang2's critical role in promoting tumor cell invasion and metastasis by stimulating cell motility and invasion mediated by the $\alpha_{\mathrm{v}} \beta_{1}$ integrin/FAK pathway in glioma cells and the $\alpha_{5} \beta_{1}$ integrin/ILK pathway in breast cancer cells independent of Tie2.

\section{Regulation of Ang2 expression}

Ang2 expression is regulated by multiple stimuli produced by tumors and their microenvironment. Tumor-derived VEGF increases Ang2 expression in tumor ECs in vivo and in vitro [20]. Insulin-like growth factor-1 and platelet-derived growth factor (PDGF) induce Ang2 expression at transcriptional and posttranslational levels in vascular smooth muscle cells [37]. Hypoxia (low oxygen tensions), which occurs frequently in human cancers, increases Ang2 mRNA expression and protein synthesis involving MAPK-, protein kinase C-, and cyclooxygenase-2-mediated pathways $[2 \bullet, 17]$. In human umbilical vein ECs, Ang2 expression also is induced by tumor necrosis factor- $\alpha$ and thrombin through distinct pathways $[38,39]$. Estrogen was shown to regulate Ang2 expression in nonreproductive tissues such as heart, kidney, and lung in vivo [40]. In adipose tissues, leptin treatment of ECs induces Ang2 expression without a concomitant increase in VEGF. The leptin upregulation of Ang2 coincided with the initiation of apoptosis in adipose ECs, suggesting a causal effect of Ang2 upregulation in adipose tissue regression [41]. Recently, Yao et al. [42] reported that high glucose regulated Ang2 transcription in mouse kidney microvascular ECs indirectly through methylglyoxal modification of a transcription cosuppressor, mSin3A, resulting in increased recruitment of O-GlcNac transferase, with consequent increased modification of Sp3. The modified Sp3 lost its capacity to bind to a glucose-responsive GC box in the Ang2 promoter, leading to increases in Ang2 expression. Additionally, the forkhead box transcription factors FOXO1 and FOXC2 specifically regulate Ang2 expression. FOXO1, one of the major FOXO factors in ECs, inhibits EC tube formation and migration. Suppression of FOXO1 by siRNA significantly downregulates expression of Ang2 as well as several other angiogenic factors in ECs, such as PDGF, leading to enhanced angiogenic activity of these cells [43]. In adipose tissues of Ang2 transgenic mice, FOXC2 overexpression transcriptionally upregulated expression of Ang2 and other angiogenic factors. Increased Ang2 switched on an angiogenic phenotype by altering vessel patterning, remodeling, and maturation of adipose vasculature. Such vascular phenotypes could be reversed when Ang2 activity was blocked by an Ang2 inhibitor in 3-week-old FOXC2 transgenic mice [44].

\section{Therapeutic Implications for Ang2}

A close link between increased Ang2 expression and tumor progression, invasion, and metastasis in various types of human cancers has established Ang2's prognostic value in predicting cancer progression and clinical outcome [17,20,21•]. Indeed, in 69 patients with acute myeloid leukemia (AML), levels of circulating Ang2 were increased by an average of 3.57-fold compared with normal controls. In a univariate Cox proportional hazards model, higher Ang2 levels predicted poor survival in AML patients [45]. In a retrospective study of 131 patients with hepatocellular carcinoma (HCC), mean Ang2 serum levels were approximately 2.3 -fold higher compared with the levels measured in 40 normal controls. The combination of Ang2 serum levels and $\alpha$-fetoprotein, which is commonly used in screening for HCC, led to improved discrimination between HCC and cirrhosis, a liver disease often associated with HCC development [46]. In 136 patients with lung cancer, Ang2 serum levels were an average of approximately 1.61-fold higher than those in 40 controls. Levels of serum Ang2 correlate with those of VEGF in patients with lung cancer, especially non-small cell lung cancer (NSCLC), but not small cell lung cancer. Significantly, NSCLC patients with distant metastases had serum Ang2 levels higher than those in patients without metastases. The 
low-Ang2 group had a better overall survival rate than the high-Ang2 group [47]. Taken together, serum Ang2 levels may be a useful clinical marker for progression of human cancers and a predictor of survival in cancer patients.

Ang2's unique roles in angiogenesis in development and progression of various types of human cancers also make Ang2 an attractive therapeutic target. Two recent studies explored such options. In a rat corneal angiogenesis model, White et al. [48] developed an iterative in vitro selection strategy called systematic evolution of ligand by exponential enrichment (SELEX) to generate nuclease-resistant RNA ligands (aptamers) that bind Ang2 with high affinity, but not other related Tie2 agonists, such as Ang1 and basic fibroblast growth factor (bFGF). This aptamer specifically inhibits the binding activity of Ang2 to Tie2 and Ang2 stimulation of tyrosine phosphorylation of Tie2. When Ang2-specific aptamer was delivered together with bFGF as bilateral corneal micro-pockets (pellets) into rat corneas, bFGF-stimulated angiogenesis in the corneas was significantly inhibited. Using various models, including in vitro ECs, tumor xenografts, and rat cornea angiogenesis, Oliner et al. [49] developed a neutralizing anti-Ang2 antibody that specifically disrupts the interaction of Ang2 and Tie2 receptor. Treatment of mice bearing xenografts of human A431 epidermoid tumor and Colo205 colon cancer using the neutralizing anti-Ang2 antibody caused static growth and eventually regression of large established tumors by suppressing cell proliferation and inducing apoptosis of tumor-derived ECs. In rats, injection of this anti-Ang2 antibody inhibited VEGF-induced corneal angiogenesis in the eye. Lastly, Cao et al. [18•] and Lee et al. [28] demonstrated that Ang2 also may be considered an inhibitor for suppression of tumor angiogenesis because systemic expression or overexpression of Ang2 attenuated VEGF expression in glioma cells expressing Ang2, impaired pericyte coverage of the tumor vasculature, inhibited EC proliferation, and induced EC apoptosis, resulting in a massive regression of tumor vasculature and tumor growth.

\section{Conclusions}

Accumulated studies on Ang2's role and therapeutic implications in angiogenesis using models of animal development, cell culture, and tumor xenografts, as well as clinical investigations, have depicted complicated functions of Ang2 in tumor angiogenesis and progression. It is well recognized that Ang2 regulates tumor angiogenesis in cooperation with VEGF as well as Ang1 through the Tie2-dependent pathways. In addition, Ang2 stimulates tumor angiogenesis, invasion, and metastasis through Tie2-independent pathways involving integrin-mediated signaling. Prognostic values of Ang2 expression in various types of human cancers and elevated Ang2 levels in the blood of cancer patients have established Ang2 as an attractive therapeutic target, and this was corroborated by recent studies using a neutralizing anti-Ang2 antibody. Ang2 also may be used to inhibit tumor angiogenesis and growth directly. However, the challenge of studying tumor angiogenesis is to understand the underlying mechanisms of the angiogenic process within the proper biologic context. As described in this article, the complexity of Ang2 functions and Ang2's regulation are highly cell context dependent. A better understanding of Ang2 functions in tumor angiogenesis and progression will lead to future advances in effective antiangiogenic and anti-cancer therapies.

\section{Acknowledgments}

The authors thank Mrs. Naama Balass for her help in proofreading this article. This work was supported in part by grants from the National Institutes of Health (CA102011 and CA130966), the American Cancer Society (RSG CSM-107144), and the Hillman Fellows Program for Innovative Cancer Research.

We apologize that because of space limitations, we could not cite as many original research articles as we would have liked. However, all the information in this report is included in several recent reviews listed in "References and Recommended Reading." 


\section{References and Recommended Reading}

Papers of particular interest, published recently, have been highlighted as:

- Of importance

- Of major importance

1. Carmeliet P, Jain RK. Angiogenesis in cancer and other diseases. Nature 2000;407:249-257. [PubMed: 11001068]

2. Shim WS, Ho IA, Wong PE. Angiopoietin: a TIE(d) balance in tumor angiogenesis. Mol Cancer Res 2007;5:655-665. [PubMed: 17634421] An excellent review of angiopoietins.

3. Davis S, Aldrich TH, Jones PF, et al. Isolation of angiopoietin-1, a ligand for the TIE2 receptor, by secretion-trap expression cloning [see comments]. Cell 1996;87:1161-1169. [PubMed: 8980223]

4. Suri C, Jones PF, Patan S, et al. Requisite role of angiopoietin-1, a ligand for the TIE2 receptor, during embryonic angiogenesis [see comments]. Cell 1996;87:1171-1180. [PubMed: 8980224]

5. Suri C, McClain J, Thurston G, et al. Increased vascularization in mice overexpressing angiopoietin-1. Science 1998;282:468-471. [PubMed: 9774272]

6. Thurston G, Suri C, Smith K, et al. Leakage-resistant blood vessels in mice transgenically overexpressing angiopoietin-1. Science 1999;286:2511-2514. [PubMed: 10617467]

7. Maisonpierre PC, Suri C, Jones PF, et al. Angiopoietin-2, a natural antagonist for Tie2 that disrupts in vivo angiogenesis [see comments]. Science 1997;277:55-60. [PubMed: 9204896]

8. Holash J, Maisonpierre PC, Compton D, et al. Vessel cooption, regression, and growth in tumors mediated by angiopoietins and VEGF. Science 1999;284:1994-1998. [PubMed: 10373119]

9. Lobov IB, Brooks PC, Lang RA. Angiopoietin-2 displays VEGF-dependent modulation of capillary structure and endothelial cell survival in vivo. Proc Natl Acad Sci U S A 2002;99:11205-11210. [PubMed: 12163646]

10. Gale NW, Thurston G, Hackett SF, et al. Angiopoietin-2 is required for postnatal angiogenesis and lymphatic patterning, and only the latter role is rescued by Angiopoietin-1. Dev Cell 2002;3:411423. [PubMed: 12361603]

11. Dellinger M, Hunter R, Bernas M, et al. Defective remodeling and maturation of the lymphatic vasculature in Angiopoietin-2 deficient mice. Dev Biol 2008;319:309-320. [PubMed: 18514180]

12. Rao S, Lobov IB, Vallance JE, et al. Obligatory participation of macrophages in an angiopoietin 2mediated cell death switch. Development 2007;134:4449-4458. [PubMed: 18039971]

13. Jones N, Iljin K, Dumont DJ, et al. Tie receptors: new modulators of angiogenic and lymphangiogenic responses. Nat Rev Mol Cell Biol 2001;2:257-267. [PubMed: 11283723]

14. Scharpfenecker M, Fiedler U, Reiss Y, et al. The Tie-2 ligand angiopoietin-2 destabilizes quiescent endothelium through an internal autocrine loop mechanism. J Cell Sci 2005;118:771-780. [PubMed: 15687104]

15. Hildbrand P, Cirulli V, Prinsen RC, et al. The role of angiopoietins in the development of endothelial cells from cord blood CD34+ progenitors. Blood 2004;104:2010-2019. [PubMed: 15213103]

16. Lemieux C, Maliba R, Favier J, et al. Angiopoietins can directly activate endothelial cells and neutrophils to promote proinflammatory responses. Blood 2005;105:1523-1530. [PubMed: 15498854]

17. Tait CR, Jones PF. Angiopoietins in tumours: the angiogenic switch. J Pathol 2004;204:1-10. [PubMed: 15307132]

18. Cao Y, Sonveaux P, Liu S, et al. Systemic overexpression of angiopoietin-2 promotes tumor microvessel regression and inhibits angiogenesis and tumor growth. Cancer Res 2007;67:3835-3844. [PubMed: 17440098] The first report suggesting that Ang2 may be used as an inhibitor to suppress tumor angiogenesis and growth by promoting vessel regression.

19. Lee OH, Fueyo J, Xu J, et al. Sustained angiopoietin-2 expression disrupts vessel formation and inhibits glioma growth. Neoplasia 2006;8:419-428. [PubMed: 16790091]

20. Bach F, Uddin FJ, Burke D. Angiopoietins in malignancy. Eur J Surg Oncol 2007;33:7-15. [PubMed: 16962282] 
21. Imanishi Y, Hu B, Jarzynka MJ, et al. Angiopoietin-2 stimulates breast cancer metastasis through the \{alpha\}5 \{beta\} 1 integrin-mediated pathway. Cancer Res 2007;67:4254-4263. [PubMed: 17483337] An excellent study demonstrating that Ang2 induces breast cancer cell motility in vitro and tumor metastasis in vivo through the integrin-mediated pathway independent of Tie2.

22. Guo P, Imanishi Y, Cackowski FC, et al. Up-regulation of angiopoietin-2, matrix metalloprotease-2, membrane type 1 metalloprotease, and laminin 5 gamma 2 correlates with the invasiveness of human glioma. Am J Pathol 2005;166:877-890. [PubMed: 15743799]

23. Hu B, Guo P, Fang Q, et al. Angiopoietin-2 induces human glioma invasion through the activation of matrix metalloprotease-2. Proc Natl Acad Sci U S A 2003;100:8904-8909. [PubMed: 12861074]

24. Eklund L, Olsen BR. Tie receptors and their angiopoietin ligands are context-dependent regulators of vascular remodeling. Exp Cell Res 2006;312:630-641. [PubMed: 16225862]

25. Kanda S, Miyata Y, Mochizuki Y, et al. Angiopoietin 1 is mitogenic for cultured endothelial cells. Cancer Res 2005;65:6820-6827. [PubMed: 16061664]

26. Cho CH, Kim KE, Byun J, et al. Long-term and sustained COMP-Ang1 induces long-lasting vascular enlargement and enhanced blood flow. Circ Res 2005;97:86-94. [PubMed: 15961719]

27. Harfouche R, Hussain SN. Signaling and regulation of endothelial cell survival by angiopoietin-2. Am J Physiol 2006;291:H1635-H1645.

28. Lee $\mathrm{OH}, \mathrm{Xu}$ J, Fueyo J, et al. Angiopoietin-2 decreases vascular endothelial growth factor expression by modulating HIF-1 alpha levels in gliomas. Oncogene 2008;27:1310-1314. [PubMed: 17704802]

29. Yancopoulos GD, Davis S, Gale NW, et al. Vascular-specific growth factors and blood vessel formation. Nature 2000;407:242-248. [PubMed: 11001067]

30. Carlson TR, Feng Y, Maisonpierre PC, et al. Direct cell adhesion to the angiopoietins mediated by integrins. J Biol Chem 2001;276:26516-26525. [PubMed: 11346644]

31. Dallabrida SM, Ismail N, Oberle JR, et al. Angiopoietin-1 promotes cardiac and skeletal myocyte survival through integrins. Circ Res 2005;96:e8-e24. [PubMed: 15692086]

32. Davis S, Papadopoulos N, Aldrich TH, et al. Angiopoietins have distinct modular domains essential for receptor binding, dimerization and superclustering. Nat Struct Biol 2003;10:38-44. [PubMed: 12469114]

33. Weber CC, Cai H, Ehrbar M, et al. Effects of protein and gene transfer of the angiopoietin-1 fibrinogen-like receptor-binding domain on endothelial and vessel organization. J Biol Chem 2005;280:22445-22453. [PubMed: 15781448]

34. Cascone I, Napione L, Maniero F, et al. Stable interaction between alpha5beta1 integrin and Tie2 tyrosine kinase receptor regulates endothelial cell response to Ang-1. J Cell Biol 2005;170:993-1004. [PubMed: 16157706]

35. Hu B, Jarzynka MJ, Guo P, et al. Angiopoietin 2 induces glioma cell invasion by stimulating matrix metalloprotease 2 expression through the $\{$ alpha $\}$ v $\{$ beta $\} 1$ integrin and focal adhesion kinase signaling pathway. Cancer Res 2006;66:775-783. [PubMed: 16424009] An excellent study demonstrating that Ang2 induces glioma cell invasion in vitro and in vivo through the integrin-FAK signaling pathway independent of Tie2.

36. Wu Y, Chen L, Zheng PS, et al. beta 1-Integrin-mediated glioma cell adhesion and free radical-induced apoptosis are regulated by binding to a C-terminal domain of PGM/versican. J Biol Chem 2002;277:12294-12301. [PubMed: 11805102]

37. Phelps ED, Updike DL, Bullen EC, et al. Transcriptional and posttranscriptional regulation of angiopoietin-2 expression mediated by IGF and PDGF in vascular smooth muscle cells. Am J Physiol Cell Physiol 2006;290:C352-C361. [PubMed: 16176970]

38. Kim I, Kim JH, Ryu YS, et al. Tumor necrosis factor-alpha upregulates angiopoietin-2 in human umbilical vein endothelial cells. Biochem Biophys Res Commun 2000;269:361-365. [PubMed: 10708557]

39. Huang YQ, Li JJ, Hu L, et al. Thrombin induces increased expression and secretion of angiopoietin-2 from human umbilical vein endothelial cells. Blood 2002;99:1646-1650. [PubMed: 11861279]

40. Ye F, Florian M, Magder SA, et al. Regulation of angiopoietin and Tie-2 receptor expression in nonreproductive tissues by estrogen. Steroids 2002;67:305-310. [PubMed: 11856554]

41. Cohen B, Barkan D, Levy Y, et al. Leptin induces angiopoietin-2 expression in adipose tissues. J Biol Chem 2001;276:7697-7700. [PubMed: 11152449] 
42. Yao D, Taguchi T, Matsumura T, et al. High glucose increases angiopoietin-2 transcription in microvascular endothelial cells through methylglyoxal modification of mSin3A. J Biol Chem 2007;282:31038-31045. [PubMed: 17670746]

43. Potente M, Urbich C, Sasaki K, et al. Involvement of Foxo transcription factors in angiogenesis and postnatal neovascularization. J Clin Invest 2005;115:2382-2392. [PubMed: 16100571]

44. Xue Y, Cao R, Nilsson D, et al. FOXC2 controls Ang-2 expression and modulates angiogenesis, vascular patterning, remodeling, and functions in adipose tissue. Proc Natl Acad Sci U S A 2008;105:10167-10172. [PubMed: 18621714]

45. Schliemann C, Bieker R, Thoennissen N, et al. Circulating angiopoietin-2 is a strong prognostic factor in acute myeloid leukemia. Leukemia 2007;21:1901-1906. [PubMed: 17597808]

46. Scholz A, Rehm VA, Rieke S, et al. Angiopoietin-2 serum levels are elevated in patients with liver cirrhosis and hepatocellular carcinoma. Am J Gastroenterol 2007;102:2471-2481. [PubMed: 17581268]

47. Park JH, Park KJ, Kim YS, et al. Serum angiopoietin-2 as a clinical marker for lung cancer. Chest 2007;132:200-206. [PubMed: 17505039]

48. White RR, Shan S, Rusconi CP, et al. Inhibition of rat corneal angiogenesis by a nuclease-resistant RNA aptamer specific for angiopoietin-2. Proc Natl Acad Sci U S A 2003;100:5028-5033. [PubMed: 12692304]

49. Oliner J, Min H, Leal J, et al. Suppression of angiogenesis and tumor growth by selective inhibition of angiopoietin-2. Cancer Cell 2004;6:507-516. [PubMed: 15542434] 